\title{
Clinical Outcomes of Posterior C2-C3 Fixation for Unstable Hangman's Fracture Compared with Posterior C1-C3 Fusion
}

\author{
Jun Hee Park, Sang Hyun Kim, Ki Hong Cho \\ Department of Neurosurgery, Ajou University School of Medicine, Suwon, Korea
}

\begin{abstract}
Objective: To verify the clinical outcomes of posterior C2-C3 fixation for unstable Hangman's fracture compared with posterior C1-C3 fixation.

Methods: Twenty four patients for unstable Hangman's fracture were enrolled between July 2007 and June 2010 in this study. Thirteen patients underwent posterior C2-C3 fusion and 11 patients underwent posterior C1-C3 fusion. Clinical outcomes were evaluated using Neck Disability Index (NDI) scores and Visual Analogue Scale (VAS) scores during preoperative and postoperative follow up period. Plain radiographs were obtained on postoperative 1 day, 1 week, and then at 1, 2, 6, and 12 months. CT was done at postoperative 12 months in all patients for evaluation of bone fusion. The mean period of clinical follow-up was 15 months.

Results: The mean ages were 43.3 years in C2-C3 group and 50.0 years in C1-C3 group. Mean follow-up period was 17.2 months in C2-C3 group and 16.3 months in C1-C3 group. VAS scores and NDI scores in C2-C3 group were much less than those in C1-C3 group at each follow-up period. The differences of VAS score and NDI scores between C2-C3 and C1-C3 groups at each follow-up period were statistically significant $(p<0.001)$ by paired T-test. Solid Bone fusion was confirmed in all cases at the final follow-up.

Conclusion: $\mathrm{C} 2-\mathrm{C} 3$ group showed better clinical and biomechanical results than C1-C3 group in terms of axial pain and disability of neck.
\end{abstract}

Key Words: Trauma $\cdot$ Spondylolisthesis $\cdot$ Fracture $\cdot$ Unstable $\cdot$ Posterior fixation

\section{INTRODUCTION}

Traumatic spondylolisthesis of the axis, or Hangman's fracture, is a common injury type of high cervical spine and the second most common fracture of axis. It involves bilateral C2 pars interarticularis fracture with a variable degree of displacement of C2 on C3 vertebrae ${ }^{8,21)}$. Although most Hangman's fractures are treated conservatively ${ }^{2,22)}$, surgery is usually preferable in cases of highly unstable Hangman's fracture and fusion failure after rigid arthrodesis ${ }^{3,5,15,21,23)}$. Surgical stabilization has been described using both anterior and posterior ap-

- Received: March 19, 2014 - Revised: May 2, 2014

- Accepted: May 30, 2014

Corresponding Author: Sang Hyun Kim, MD, PhD

Department of Neurosurgery Ajou University Hospital, 164, World Cup-ro,

Yeongtong-Gu, Suwon, Kyunggi-do 443-380, Korea

Tel: +82-31-219-5230, Fax: +82-31-219-5238

E-mail: kaliemd@yahoo.com/shkim709@ajou.ac.kr

$\otimes T h i s$ is an Open Access article distributed under the terms of the Creative

Commons Attribution Non-Commercial License (http://creativecommons.org/ licenses/by-nc/3.0/) which permits unrestricted non-commercial use, distribution, and reproduction in any medium, provided the original work is properly cited. proaches $^{17,20-21,26-27)}$. Among the different surgical fixation techniques, posterior screw and rod fixation can offer better results in terms of alignment and stabilization of spine with a low level of pseudoarthrosis than those of anterior plate and screw fixation because anterior approach does not address the detached posterior arch of $\mathrm{C} 2$ and instability remained during flexion-extension and axial rotation ${ }^{12}$. This technique addresses the detached posterior arch of $\mathrm{C} 2$ by pinning the fractured pars while simultaneously addressing instability at the disc by immobilizing $\mathrm{C} 2$ relative to $\mathrm{C} 3$.

Posterior C1-C3 lateral mass screw and rod fixation can provide good alignment and firm stabilization of spine. However, this technique cannot preserve the motion at intact C1-C2 segment. Posterior $\mathrm{C} 2$ pedicle - $\mathrm{C} 3$ lateral mass screw and rod fixation can be considered to preserve the motion of the axis in cases that direct repair of the pars fracture with a screw across the fracture line is possible ${ }^{3,5,23)}$. The key difference between these two techniques is preservation of C1-C2 motion which may have difference in biomechanical features as well as clinical outcomes. This study was conducted to verify the clinical outcomes of posterior C2-C3 fixation for unstable Hangman's fracture compared with posterior C1-C3 fixation. 


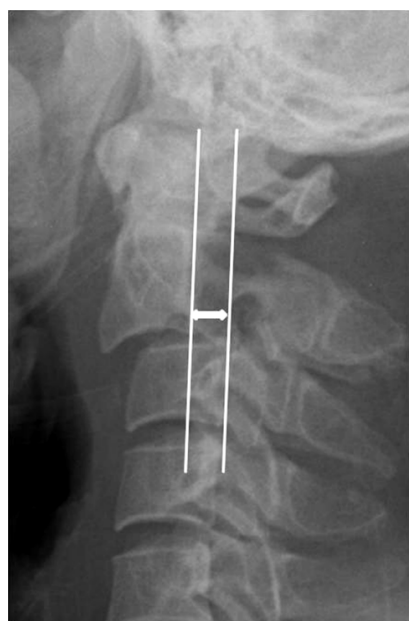

Fig. 1. Measurement of anterior translation. The distance between parallel lines drawn through posterior borders of $\mathrm{C} 2$ and C3 vertebral bodies.

\section{MATERIALS AND METHODS}

Twenty four consecutive patients with unstable Hangman's fracture were enrolled who required posterior surgical fixation and stabilization using the Polyaxial Screw-rod System (Synapse, Bettlach and Waldenburg, Switzerland) between July 2007 and June 2010. Thirteen patients underwent posterior $\mathrm{C} 2-\mathrm{C} 3$ fixation (C2-C3 group) and 11 patients underwent posterior C1-C3 fixation (C1-C3 group). The medical records of all patients were reviewed. All patients were classified and selected using Classification of hangman's fracture which was proposed by Levine and Edwards to evaluate ${ }^{16-17)}$. Type I has stable and minimal translation $(<3 \mathrm{~mm})$ without $\mathrm{C} 2-\mathrm{C} 3$ angulation. Type II has unstable disc disruption and significant C2-C3 angulation and translation ( $>3 \mathrm{~mm}$ ). Type IIA is unstable due to flexion-distraction injury and has more angulation than type 2 without translation. Type III is unstable and has severe $\mathrm{C} 2-\mathrm{C} 3$ angulation and translation. It has sometimes unilateral or bilateral facet dislocation. Surgical stabilization was recommended in Levine-Edwards Type IIa and III fractures with significant dislocation. Surgical treatments were performed in patient who showed poor reduction or nonunion after rigid external orthosis, neurologic deficit or unstable combination fracture at the presentation.

Dynamic plain radiographs were obtained on postoperative day 1,1 week, and then at 1, 2, 6, and 12 months and Computed Tomographic (CT) evaluation was done at postoperative 12 months in all patients for evaluation of spinal stability and bone fusion. The adequacy of fusion was determined at approximately four, six and 12 months postoperatively. Pseudoarthrosis was defined as a motion $>2.0 \mathrm{~mm}$ between the laminae at the base of the spinous processes of the upper and lowermost fixed and fused levels on the flexion/extension plain radiographs.

Concurrently, clinical outcomes were evaluated using extent of anterior translation, rotation, Visual Analogue Scale (VAS) scores, and Neck Disability Index (NDI) scores during preoperative and every postoperative follow up visit ${ }^{24)}$.

Extent of anterior translation was measured as the distance between parallel lines drawn through the posterior borders of $\mathrm{C} 2$ and $\mathrm{C} 3$ vertebral bodies (Fig. 1). Range of rotation was measured at final follow up visit when patients turned their neck to look over the both shoulders in a supine position. Postoperative pain was assessed using a 10-point VAS with endpoint anchors of no pain ( 0 points) and severe pain (10 points). The NDI questionnaire is comprised of 10 single items related to activities of daily living. Each item has six predefined response categories, coded 0-5 on an ordinal scale. The scores reflected either the degree of neck pain or the degree of difficulty in performing certain actions due to neck pain. The lowest score (0) represented no problem or pain, whereas the highest score (5) represented maximum problems or pain. Patients who underwent anterior fixation or combined anterior and posterior fixation were excluded. The pre- and postoperative arm and neck symptom VAS scores and NDI scores were compared using two-sample $t$ tests paired for means. A $\mathrm{p}$ value of $<0.05$ was regarded as significant.

Inclusion criteria for posterior $\mathrm{C} 2-\mathrm{C} 3$ fixation included as below; linear fracture without bony particles in a gap of fracture, $\leq 5 \mathrm{~mm}$ of displacement between $\mathrm{C} 2$ and $\mathrm{C} 3, \leq 11$ degree of angulation between $\mathrm{C} 2$ and $\mathrm{C} 3$, and no injury of $\mathrm{C} 1$. Posterior C1-C3 fixation had been perform in cases with communited fracture of $\mathrm{C} 2$ isthmus, $>6 \mathrm{~mm}$ of displacement between $\mathrm{C} 2$ and $\mathrm{C} 3,>12$ degree of angulation between $\mathrm{C} 2$ and $\mathrm{C} 3$, narrow or cortical pedicle of $\mathrm{C} 2$, and existence of C1 injury or C1-C2 instability (Fig. 2).

\section{Surgical Technique}

The surgery was performed in a consistent fashion. A standard midline incision was made above the C1-C4 levels. The lateral masses were exposed in a subperiosteal fashion to the lateral margins of the facet joints. Once the displacement was reduced, the lateral masses to be fused were decorticated with the drill. Great care was taken not to disturb the capsule at adjacent levels in order to prevent any iatrogenic instability.

Pedicle screws were used at C2 vertebra and lateral mass screws were used at $\mathrm{C} 3$ vertebra under the $\mathrm{C}$-arm guidance for C2-C3 fixation (Fig. 3A). Lateral mass screws were used at $\mathrm{C} 1$ and pedicle screws or lateral mass screws were used at C3 vertebra for C1-C3 fixation (Fig. 3B). Pedicle screw insertion for $\mathrm{C} 2$ vertebra was done unilaterally or bilaterally as possible. A rod was prepared to the appropriate length 
and contour that it would easily pass through the heads of all polyaxial screws. Once the rod was positioned, it was secured to the heads of the screws using outer nuts.

Bony fusion was performed by packing allograft bone and
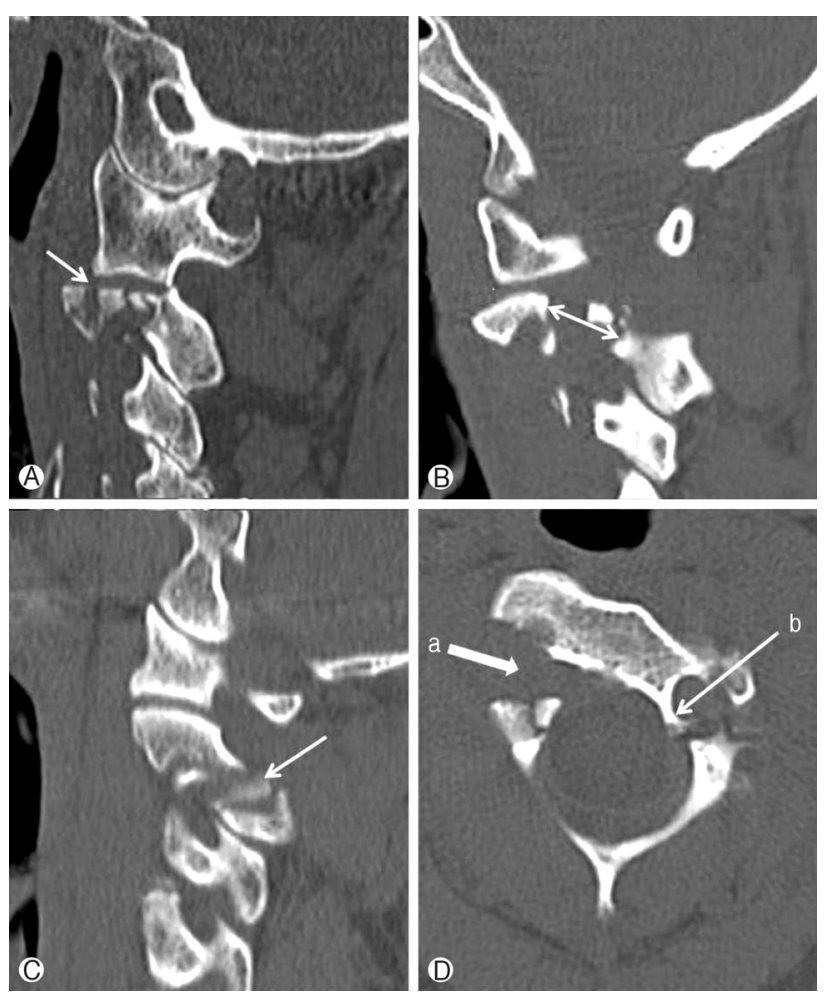

Fig. 2. Inclusion criteria for posterior $\mathrm{Cl}-\mathrm{C} 3$ fixation. (A) communited fracture of C2 isthmus, (B) $>6 \mathrm{~mm}$ of displacement between C2 and $\mathrm{C} 3,(\mathrm{C})$ bony particles in a gap of fracture, (D-a) $>12$ degree of angulation between C2 and C3, (D-b) narrow or cortical pedicle of $\mathrm{C} 2$, and existence of $\mathrm{Cl}$ injury or $\mathrm{Cl}-\mathrm{C} 2$ instability.
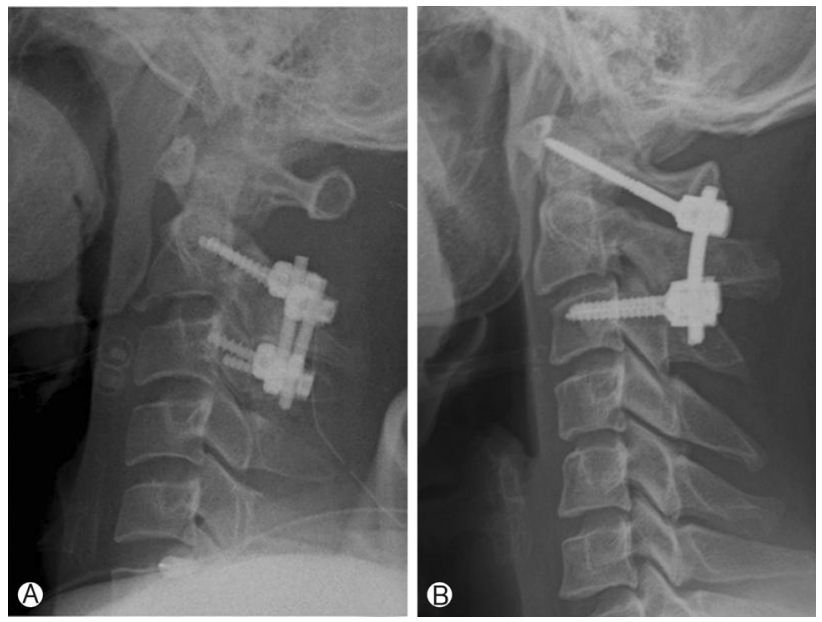

Fig. 3. Postoperative $\mathrm{C}$-spine lateral views for posterior $\mathrm{C} 2-\mathrm{C} 3$ fixation (A) and $\mathrm{Cl}-\mathrm{C} 3$ fixation $(\mathrm{B})$. demineralized bone matrix (DBM) into the facet joints, around the decorticated lateral masses lateral to the rod and laminae. Bone graft was placed between the posterior arch of $\mathrm{C} 1$ and the lamina-spinous process of $\mathrm{C} 2$ vertebra after $\mathrm{C} 1-\mathrm{C} 3$ fixation. During the entire procedure, the retractors were intermittently released to avoid denervation of the erector spinal muscles. Routine closure was carried out and drains were left in place as needed.

\section{RESULTS}

Twenty four patients were enrolled in this study. Demographic and clinical data of the patients were shown in Table 1. C2-C3 group consisted of 8 men and 5 women whose mean age was 43.3 years (range 29-57). C1-C3 group consisted of 6 men and 5 women whose mean age was 50.0 years (range 26-68). All patients were regularly seen in the follow-up clinic at periodic interval after the procedure. Mean follow-up period was 17.2 months in C2-C3 group and 16.3 months in C1-C3 group. Solid bone fusion was confirmed in all cases at postoperative 1 year follow-up on both $\mathrm{C} 2-\mathrm{C} 3$ and $\mathrm{C} 1-\mathrm{C} 3$ fixation groups (Fig. 4).

The average translations were $4.7 \pm 1.16$ in C2-C3 group and $8.54 \pm 2.34$ in C1-C3 group at presentation. The average translations were $1.2 \pm 1.02$ in C2-C3 group and $2.3 \pm 1.57$ at final follow-up. Although degree of reduction showed much

Table 1. Demographic and clinical data of the patients based on procedures

\begin{tabular}{lll}
\hline \hline \multicolumn{1}{c}{ Characteristics } & C2-C3 fixation & C1-3fixation \\
\hline Number of patient & 13 & 11 \\
Male : Female & $8: 5$ & $6: 5$ \\
Age (year) & 43.3 & 50.0 \\
& (range18-69) & (range 26-68) \\
Duration of follow-up (month) & 17.2 & 16.3 \\
& (range12-24) & (range 2-24) \\
ROM for neck & & \\
VAS score (mean) & & \\
Postoperative 1 day (1 day) & 8.38 & 9.45 \\
Postoperative 1 month (1 mo) & 4.07 & 6.18 \\
Postoperative 3 month (3 mo) & 2.69 & 5.27 \\
Postoperative 6 month (6 mo) & 2.15 & 4.27 \\
Final follow-up & 2.07 & 3.45 \\
NDI & & \\
Postoperative 1 day (1 day) & 84.15 & 89.09 \\
Postoperative 1 month (1 mo) & 55.38 & 76.18 \\
Postoperative 3 month (3 mo) & 32.31 & 64.72 \\
Postoperative 6 month (6 mo) & 24.31 & 46.90 \\
Final follow-up & 22.61 & 44.18 \\
\hline
\end{tabular}




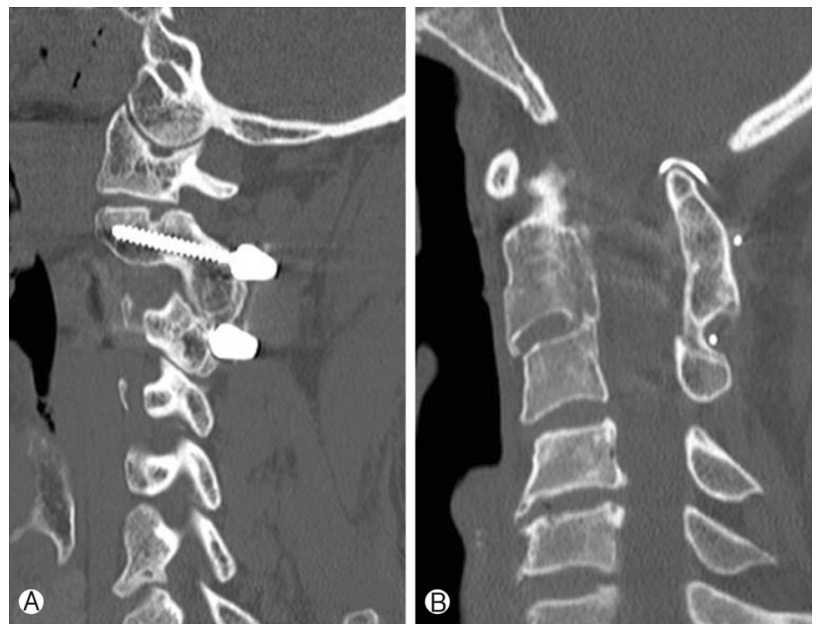

Fig. 4. Solid bone fusion was confirmed in all cases at postoperative 1 year on both posterior $\mathrm{C} 2-\mathrm{C} 3$ fixation $(\mathrm{A})$ and posterior $\mathrm{C}$ - $-\mathrm{C} 3$ fixation (B)

higher in C1-C3 group, restoration to normal cervical alignment could be achieved in C2-C3 group compared with C1C3 group at final follow-up.

The average ranges of rotation were $120 \pm 6.15^{\circ}$ in C2-C3 group and $19.8 \pm 3.54^{\circ}$ in C1-C3 group at final follow-up.

In C2-C3 group, the average VAS score for neck pain was $8.3 \pm 1.11$ day after the surgery. VAS score was gradually decreased during the follow-up period and the mean VAS score was decreased up to $2.07 \pm 0.8$ at final follow-up (Fig. 5). In C1-C3 group, the average VAS score for neck pain was 9.45 \pm 0.51 day after the surgery. VAS score was slowly decreased during the follow-up period and the mean VAS score was decreased up to $3.45 \pm 0.7$ at final follow-up. VAS scores in C2-C3 group were much less than those in C1-C3 group at each follow-up period. The differences of VAS score between C2-C3 and C1-C3 groups at each follow-up period were statistically significant $(\mathrm{p}<0.001)$ by paired T-test.

In C2-C3 group, the average NDI score was $84.1 \pm 2.6$ 1 day after the surgery. NDI score was gradually decreased during the follow-up period and the mean NDI score was decreased up to $22.6 \pm 2.8$ at final follow-up (Fig. 6). In C1-C3 group, the average NDI score was $89.0 \pm 2.11$ day after the surgery. NDI score was slowly decreased during the follow-up period and the mean NDI score was decreased up to 44.1 \pm 5.1 at final follow-up. NDI scores in C2-C3 group were much less than those in $\mathrm{C} 1-\mathrm{C} 3$ group at each follow-up period. The differences of NDI score between C2-C3 and C1-C3 groups at each follow-up period were statistically significant $(\mathrm{p}<0.001)$ by paired T-test.

There were one case of wound infection and 1 case of screw loosening in C2-C3 group. Revision surgery for screw repositioning was needed in case of screw loosening. There was

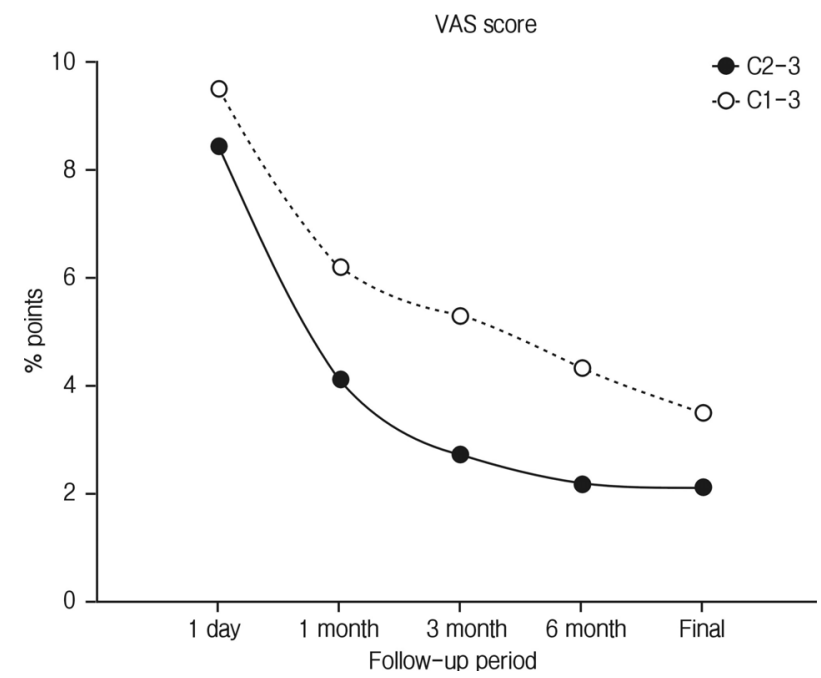

Fig. 5. The postoperative change of VAS scores for neck pain. VAS scores in C2-C3 group were much less than those in C1-C3 group at each follow-up period. The differences of VAS score between C2-C3 and C1-C3 groups at each follow-up period were statistically significant $(p<0.001)$ by paired T-test, except immediate postoperative period.

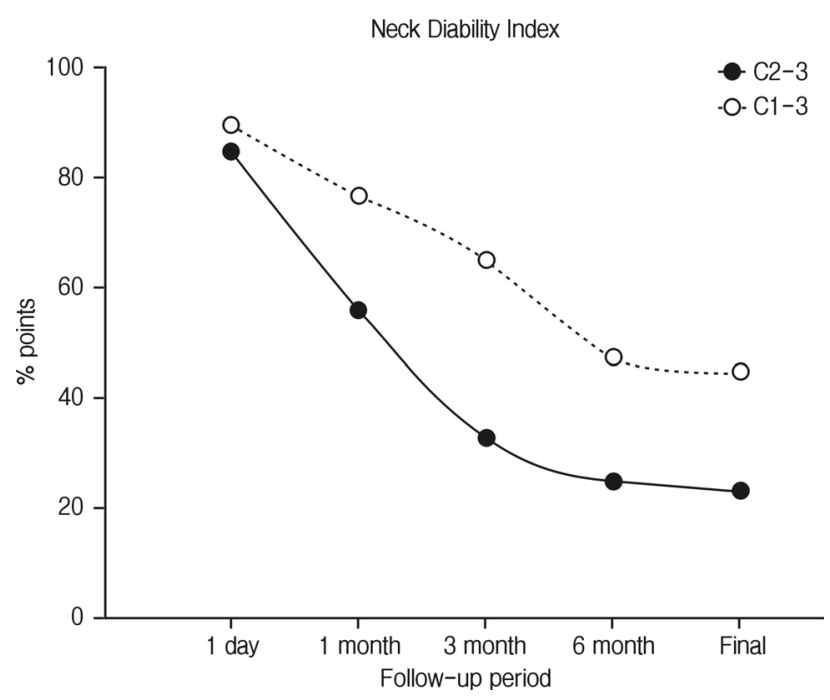

Fig. 6. The postoperative change of NDI scores. NDI scores in C2-C3 group were much less than those in $\mathrm{Cl}-\mathrm{C} 3$ group at each follow-up period. The differences of NDI score between C2-C3 and C1-C3 groups at each follow-up period were statistically significant $(p<0.001)$ by paired T-test.

no complication in C1-C3 group.

\section{DISCUSSION}

Hangman's fracture is the second most common fracture of the second cervical vertebra (C2). It involves a bilateral 
arch fracture of the $\mathrm{C} 2$ pars interarticularis with variable displacement of $\mathrm{C} 2$ on $\mathrm{C}^{7}$. Among different classifications for Hangman's fracture, Levine-Edward's is the most frequently used $^{8-10,16}$. Type I lesion is usually considered stable with no angulation and displacement on C2-C3, whereas Types II, IIa, and III are usually considered unstable. These unstable types are combined with the injury of the $\mathrm{C} 2-\mathrm{C} 3$ disc and the anterior longitudinal ligament. In Type II and type III Hangman's fracture, extension forces have been implicated in the disruption of the anterior longitudinal ligament, posterior longitudinal ligament, and $\mathrm{C} 2-\mathrm{C} 3$ disc.

In most cases of Hangman's fracture, the conservative treatment is the most indicated ${ }^{4,6,9,22-23,25)}$. Semirigid collars are used for cases with a small degree of dislocation. Rigid immobilizations such as halo-vest are used for cases of great displacement. In regard to the unstable Hangman's fracture, the treatment is still controversial. Pseudoarthrosis, anterior dislocation, angulation of $\mathrm{C} 2$ over $\mathrm{C} 3$, and recurrent axial pain were observed in about $60 \%$ of the cases of Types II, IIa, and III, which were primarily treated with conservative therapy ${ }^{9-10,22)}$. Majority of authors recently suggested that the presence of discoligamentous injury on top of bony injury would require surgery. Another reason for surgery is the shortening the course of treatment ${ }^{3-4,18,21,23,26-27)}$.

The goals in the surgical treatment of Type II and III Hangman's fractures are reduction, stabilization, and maintenance of alignment. Although method of surgical stabilization is selected according to severity of $\mathrm{C} 2$ fracture and degree of displacement, specific anatomical aspect of atlantoaxial complex should be also considered $\mathrm{C} 2$ vertebra is designed to provide a bearing surface on which the atlas may rotate and pars interarticularis is most stressed by translational movement between adjacent segments in cervical spine ${ }^{7-8)}$. Surgical stabilization has been described in both anterior and posterior approaches. Among the different posterior approaches, direct repair of the pars fracture with a screw across the fracture line has the advantage of preserving motion of the axis ${ }^{3,5,17)}$. However, direct pars repair does not address instability at the disc. The main advantage of this technique is not having to sacrifice any normal motion of the $\mathrm{C} 2$ segment and fixating only the fractured bones of $\mathrm{C} 2$. However, it can be used only in cases with minimal or no C2-C3 disc injury. The application of posterior C2 and $\mathrm{C} 3$ fixation to a highly unstable hangman's fracture results in the aggravation of the forward displacement of $\mathrm{C} 2$ due to the intraoperative prone position, particularly with the extremely unstable state of $\mathrm{C}^{5,11,17-18,27)}$. This phenomenon can cause iatrogenic injury and may lead to extremely negative consequences. Posterior C1-C3 wiring techniques have also been described, these techniques require postoperative halothoracic immobilization ${ }^{4)}$. Anterior C2-C3 interbody fusion was currently attempted first, followed by the procedure involving direct one-stage posterior $\mathrm{C} 2$ and $\mathrm{C} 3$ pedicle screw fixation in the fracture at the isthmus position ${ }^{18)}$. Two separate incision and fusion surgery is needed, although it seems to provide adequate spinal immobilization.

Polyaxial screw and rod fixation system has been developed and is widely used as a standard instrument. Posterior C1-C3 lateral mass screws and rods fixation has been performed with satisfactory fusion rate ${ }^{6,13-14)}$. Recent studies have also reported that the biomechanical comparison of stabilization techniques on Hangman's fracture and posterior C2-C3 screw and rod construction was found to be more effective on the stabilization of Hangman's fracture than anterior cervical plating and C2 pars screwing ${ }^{7}$. Pedicle screw fixation is a biomechanically stronger repair method and requires only a posterior approach $^{1,19)}$. In addition, pedicle screw instrumentation through a single stage posterior approach offered better biomechanical stability in 3 column spinal injury. However, pedicle screw placement is technically demanding because of large individual variations in the pedicle dimensions and course of the vertebral artery.

Both posterior C1-C3 and C2-C3 fixation can offer high fusion rate. However, posterior C1-C3 fixation immobilized intact C1-C2 segment and leaded serious limitation of neck motion and axial pain. This showed the differences in clinical outcomes between these two techniques. In C2-C3 group, the average VAS score for neck pain and NDI scores were much less than those in C1-C3 group right after the surgery and these differences continued until final follow-up. The differences of VAS score and NDI score between C2-C3 and C1-C3 groups at each follow-up period were statistically significant $(p<0.001)$ by paired T-test. Even though other clinical situations, such as accompanying injury to soft tissue or other levels and severity of dislocation, these differences of VAS score and NDI score at final follow-up visit with the full recovery from the initial injury can show clinical significance. C2-C3 group showed better clinical and biomechanical results than $\mathrm{C} 1-\mathrm{C} 3$ group in terms of axial pain and disability of neck.

There was one case of screw loosening in C2-C3 group which needed revision surgery for screw repositioning. Although posterior C2-C3 fixation for unstable Hangman's fracture offers biomechanical stability without additional treatment, there are some prerequisite conditions for achievement of good clinical and radiological results. Complete reduction and close adhesion of fracture gap should be achieved before the insertion of $\mathrm{C} 2$ screw. And, we have to press down the screw during insertion of $\mathrm{C} 2$ pedicular screw not to make fracture gap wide. Even if complete reduction is not achieved before the insertion of pedicle screws, bicortical purchase of $\mathrm{C} 2$ pedicular screw, or posterior C1-C3 fixation, is recommended. 
Even though posterior C2-C3 fixation for unstable Hangman's fracture may have better clinical outcomes compared with those of posterior C1-C3 fixation, clinical application should be carefully considered regarding severity of injury of $\mathrm{C} 2$ vertebra and instability to adjacent vertebrae.

\section{CONCLUSION}

A higher degree of immobilization and strong fixation may lead higher fusion rate of hangman's fracture. Even though higher and satisfactory fusion rate can be achieved in both posterior $\mathrm{C} 2-\mathrm{C} 3$ fixation and $\mathrm{C} 1-\mathrm{C} 3$ fixation groups, their clinical outcomes can be different because of pain and neck disability. Posterior C2-C3 fixation is a segmental fixation in patient with Hangman's fracture and may be feasible surgical treatment in proper indication compared with posterior C1-C3 fixation.

\section{REFERENCES}

1. Abumi, Shono, Ito, Taneichi, Kotani, Kaneda: Complications of pedicle screw fixation in reconstructive surgery of the cervical spine. Spine (Phila Pa 1976) 25:962-969, 2000

2. Arand, Neller, Kinzl, Claes, Wilke: The traumatic spondylolisthesis of the axis. A biomechanical in vitro evaluation of an instability model and clinical relevant constructs for stabilization. Clin Biomech (Bristol, Avon) 17:432-438, 2002

3. Borne, Bedou, Pinaudeau: Treatment of pedicular fractures of the axis. A clinical study and screw fixation technique. J Neurosurg 60:88-93, 1984

4. Bridwell: Treatment of a markedly displaced hangman's fracture with a luque rectangle and a posterior fusion in a 71-year-old man. Case report. Spine (Phila Pa 1976) 11:49-52, 1986

5. Bristol, Henn, Dickman: Pars screw fixation of a hangman's fracture: technical case report. Neurosurgery 56:E204; discussion E204, 2005

6. Coric, Wilson, Kelly: Treatment of traumatic spondylolisthesis of the axis with nonrigid immobilization: a review of 64 cases. J Neurosurg 85:550-554, 1996

7. Duggal, Chamberlain, Perez-Garza, Espinoza-Larios, Sonntag, Crawford: Hangman's fracture: a biomechanical comparison of stabilization techniques. Spine (Phila Pa 1976) 32:182-187, 2007

8. Effendi, Roy, Cornish, Dussault, Laurin: Fractures of the ring of the axis. A classification based on the analysis of 131 cases. J Bone Joint Surg Br 63-B:319-327, 1981
9. Fielding, Francis, Hawkins, Pepin, Hensinger: Traumatic spondylolisthesis of the axis. Clin Orthop Relat Res:47-52, 1989

10. Francis, Fielding: Traumatic spondylolisthesis of the axis. Orthop Clin North Am 9:1011-1027, 1978

11. Greene, Dickman, Marciano, Drabier, Hadley, Sonntag: Acute axis fractures. Analysis of management and outcome in 340 consecutive cases. Spine (Phila Pa 1976) 22:1843-1852, 1997

12. Hadley, Browner, Sonntag: Axis fractures: a comprehensive review of management and treatment in 107 cases. Neurosurgery 17:281-290, 1985

13. Hadley, Dickman, Browner, Sonntag: Acute axis fractures: a review of 229 cases. J Neurosurg 71:642-647, 1989

14. Horn, Hott, Porter, Theodore, Papadopoulos, Sonntag: Atlantoaxial stabilization with the use of C1-3 lateral mass screw fixation. Technical note. J Neurosurg Spine 5:172-177, 2006

15. Karimi-Nejad: Surgical management of cervical spine injuries. Neurosurg Rev 12 Suppl 1:525-535, 1989

16. Levine, Edwards: The management of traumatic spondylolisthesis of the axis. J Bone Joint Surg Am 67:217-226, 1985

17. Li, Dai, Lu, Chen: A systematic review of the management of hangman's fractures. Eur Spine J 15:257-269, 2006

18. Liu, Li, Wu: One-stage posterior $\mathrm{C} 2$ and $\mathrm{C} 3$ pedicle screw fixation or combined anterior C2-C3 fusion for the treatment of unstable hangman's fracture. Exp Ther Med 5:667-672, 2013

19. Ludwig, Kowalski, Edwards, Heller: Cervical pedicle screws: comparative accuracy of two insertion techniques. Spine (Phila Pa 1976) 25:2675-2681, 2000

20. Muller, Wick, Muhr: Traumatic spondylolisthesis of the axis: treatment rationale based on the stability of the different fracture types. Eur Spine J 9:123-128, 2000

21. Tuite, Papadopoulos, Sonntag: Caspar plate fixation for the treatment of complex hangman's fractures. Neurosurgery 30:761764; discussion 764-765, 1992

22. Vaccaro, Madigan, Bauerle, Blescia, Cotler: Early halo immobilization of displaced traumatic spondylolisthesis of the axis. Spine (Phila Pa 1976) 27:2229-2233, 2002

23. Verheggen, Jansen: Hangman's fracture: arguments in favor of surgical therapy for type II and III according to Edwards and Levine. Surg Neurol 49:253-261; discussion 261-252, 1998

24. Vernon, Mior: The Neck Disability Index: a study of reliability and validity. J Manipulative Physiol Ther 14:409-415, 1991

25. Wilson: Treatment of traumatic spondylolisthesis of the axis with nonrigid immobilization: erratum. J Neurosurg 85:1198, 1996

26. Wilson, Marshall, Ewart: Transoral fusion with internal fixation in a displaced hangman's fracture. Spine (Phila Pa 1976) 24: 295-298, 1999

27. Ying, Wen, Xinwei, Yong, Hongyu, Zhu, et al: Anterior cervical discectomy and fusion for unstable traumatic spondylolisthesis of the axis. Spine (Phila Pa 1976) 33:255-258, 2008 